\title{
An uncommon complication of infective endocarditis
}

\author{
KW Lam *, KW Au Yeung, KY Lai
}

Hong Kong Med J 2015;21:187.e1-2

DOI: $10.12809 / \mathrm{hkmj} 144248$

A 20-year-old man presented to us with confusion and generalised skin rash in April 2010. On examination, he was febrile and in shock. He was detected with a mitral regurgitation murmur and mild neck stiffness. His white cell count was elevated and platelet count was low. His liver function was mildly impaired. Chest X-ray showed acute pulmonary oedema. Computed tomography (CT) scan of brain showed
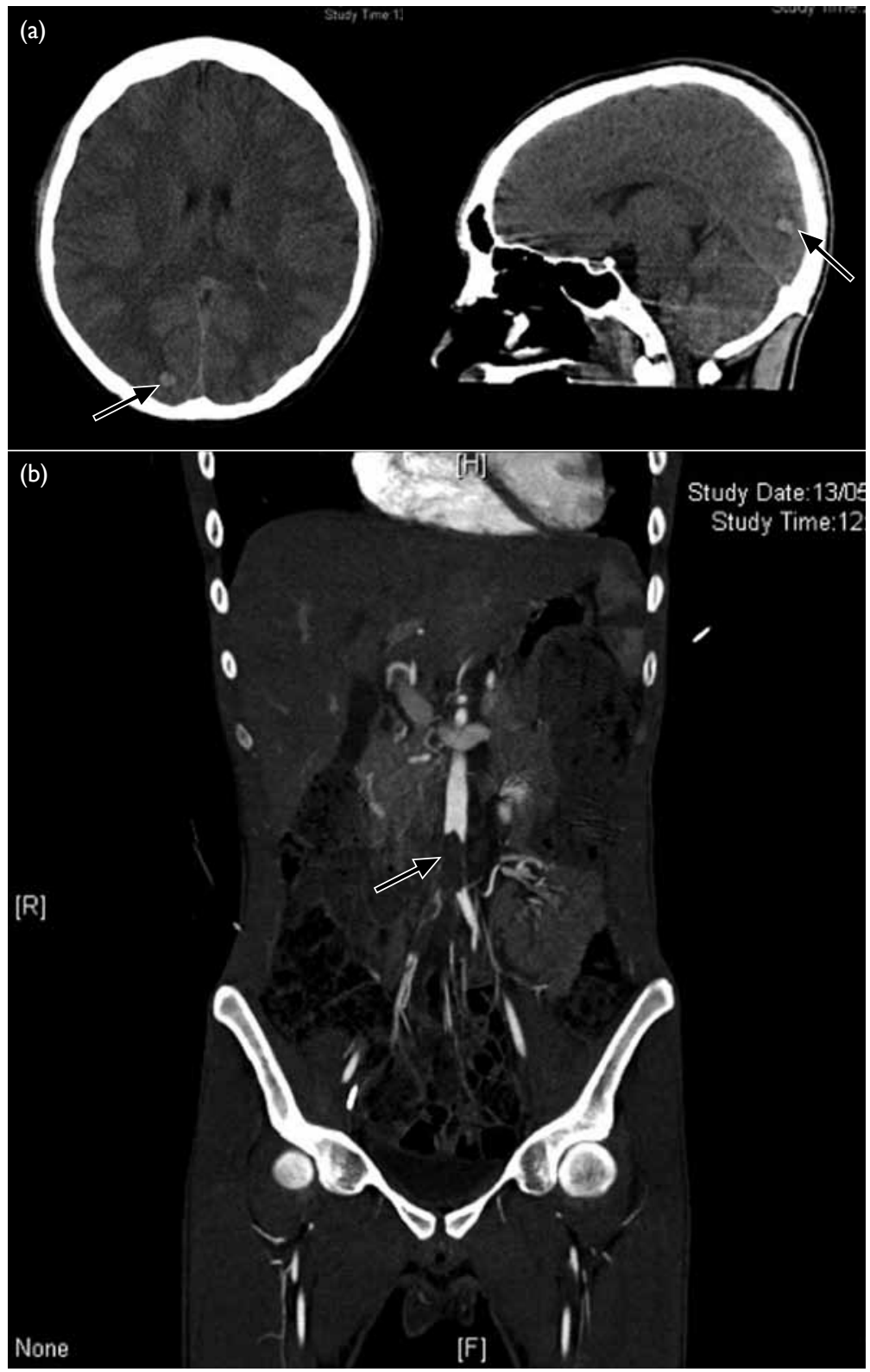

FIG I. Complications of infective endocarditis developed by the patient: (a) cerebral haemorrhage in the left occipital lobe (arrows), (b) saddle embolism (arrow) multiple haematomas in his left occipital lobe and right parietal lobe, and subarachnoid haemorrhage (Fig 1a). Blood culture showed methicillin-sensitive Staphylococcus aureus (MSSA).

Transthoracic echocardiogram showed a huge vegetation measuring $4 \mathrm{~cm} \mathrm{x} 2.22 \mathrm{~cm}$ attached to the base of anterior leaflet of mitral valve, resulting in perforation of the base of leaflet with severe mitral regurgitation (Fig 2a). The diagnosis was infective endocarditis due to MSSA, complicated by ruptured chordae tendineae with severe acute mitral regurgitation and multiple, septic cerebral emboli. The source of infection was suspected to be the skin. He was treated with high-dose intravenous cloxacillin and gentamicin. His heart failure was treated with frusemide.

Three weeks later, he complained of sudden onset of right lower limb pain and numbness. His CT angiogram showed a large saddle embolus in the lower abdominal aorta (Fig 1b). Emergent right-sided lower femoral and bilateral iliac artery embolectomy was performed.

On postoperative echocardiogram, the size of the vegetation was found to be decreased to $1.5 \mathrm{~cm}$ in diameter (Fig 2b). He then underwent mitral valvular replacement about 1 week later.

\section{Discussion}

The incidence of community-acquired native-valve endocarditis in western countries ranges from 1.7 to 6.2 cases per 100000 person-years, with a maleto-female ratio of $1.7: 1{ }^{1}$ Recently, the incidence of $S$ aureus infective endocarditis is on the rise. Infective endocarditis due to $S$ aureus is more common among young adults, especially the intravenous injection drug users. ${ }^{1}$ Usually, the tricuspid valve is involved. ${ }^{2}$

Extra cardiac complications of infective endocarditis usually include embolic events. The rate of embolic events has a relationship with the initiation of antibiotic therapy. In one study, after the commencement of appropriate antimicrobial treatment, the rate of embolism fell from 13 per 1000 patient-days during the first week of treatment to fewer than 1.2 per 1000 patient-days 2 weeks after treatment. $^{3}$ A review involving 281 patients with suspected infective endocarditis demonstrated that the incidence of embolic events was greater with mitral than aortic valve vegetations (25\% vs $10 \%) .{ }^{4}$ For mitral valve vegetations, the rate of embolism was higher if these were attached to the anterior leaflet 


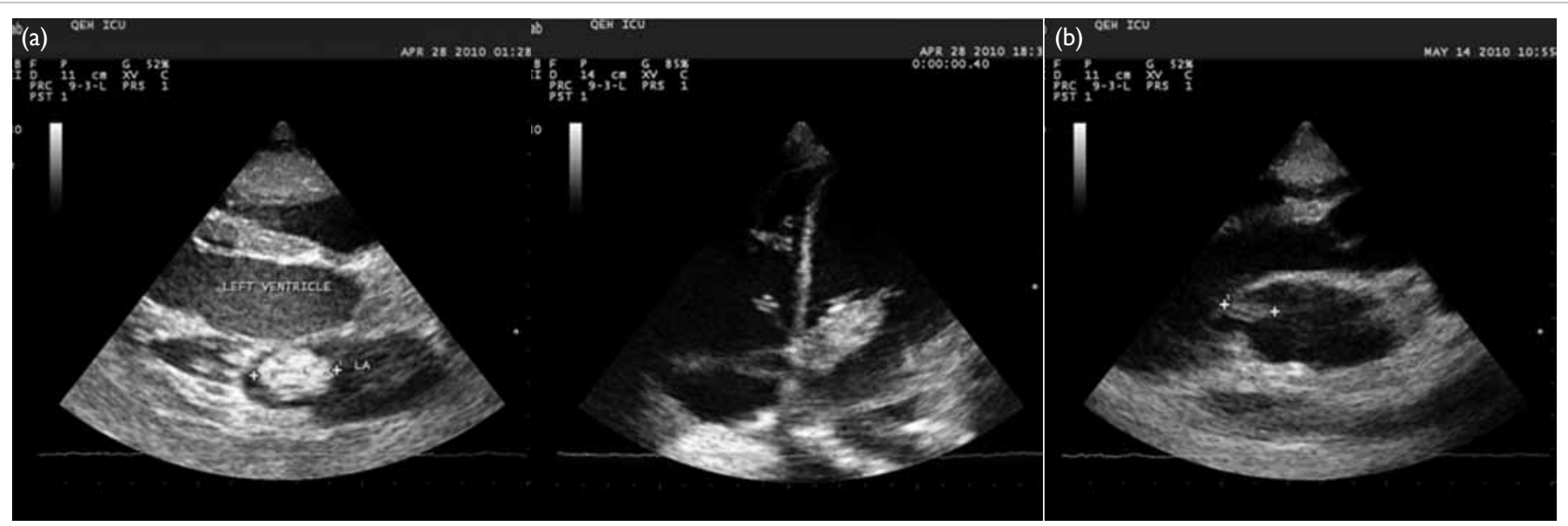

FIG 2. Echocardiographic findings in the vegetation (a) before and (b) after embolisation

rather than posterior leaflet. Some studies showed that the rate of embolism correlated with the size of vegetation, with the risk being higher if the diameter of the vegetation was greater than $1 \mathrm{~cm} .{ }^{1}$

Up to $65 \%$ of embolic events of infective endocarditis are associated with neurological involvement. Such neurological complications account for $20 \%$ to $40 \%$ of all patients with infective endocarditis. ${ }^{5}$ Saddle embolus is a large embolus that straddles the arterial bifurcation and, thus, blocks both branches of the aorta. Saddle embolisation at the aortic bifurcation is an uncommon but serious complication. From the literature search, only eight cases were reported and all were caused by fungal endocarditis. ${ }^{6}$

KW Lam *, MB, BS, FHKAM (Medicine)

KW Au Yeung, MB, BS, FHKAM (Anaesthesiology)

KY Lai, MB, BS, FHKAM (Medicine)

Intensive Care Unit, Queen Elizabeth Hospital, Jordan, Hong Kong

* Corresponding author: lamkw1@ha.org.hk

\section{References}

1. Mylonakis E, Calderwood SB. Infective endocarditis in adults. N Engl J Med 2001;345:1318-30.

2. Hecht SR, Berger M. Right-sided endocarditis in intravenous drug users. Prognostic features in 102 episodes. Ann Intern Med 1992;117:560-6.

3. Heiro M, Nikoskelainen J, Engblom E, Kotilainen E, Marttila R, Kotilainen P. Neurologic manifestations of infective endocarditis: a 17-year experience in a teaching hospital in Finland. Arch Inern Med 2000;160:2781-7.

4. Rohmann S, Erbel R, Görge G, et al. Clinical relevance of vegetation localization by transoesophageal echocardiography in infective endocarditis. Eur Heart J 1992;13:446-52.

5. Røder BL, Wandall DA, Espersen F, Frimodt-Møller N, Skinhøj P, Rosdahl VT. Neurologic manifestations in Staphylococcus aureus endocarditis: a review of 260 bacteremic cases in nondrug addicts. Am J Med 1997;102:379-86.

6. Kawamoto T, Nakano S, Matsuda H, Hirose H, Kawashima Y. Candida endocarditis with saddle embolism: a successful surgical intervention. Ann Thorac Surg 1989;48:723-4. 